\title{
Endocrine response after prior treatment with fulvestrant in postmenopausal women with advanced breast cancer: experience from a single centre
}

\author{
$K L$ Cheung, $R$ Owers and J F R Robertson \\ Division of Breast Surgery, University of Nottingham, Nottingham City Hospital, Hucknall Road, Nottingham NG5 1PB, UK \\ (Requests for offprints should be addressed to K L Cheung; Email: kl.cheung@nottingham.ac.uk)
}

\begin{abstract}
The pure anti-oestrogen fulvestrant has now been licensed for use in advanced breast cancer which has progressed on an anti-oestrogen. Optimal sequencing of various endocrine agents becomes very important in the therapeutic strategy. We report our experience of further endocrine response with another endocrine agent after prior fulvestrant treatment. Among all patients with advanced breast cancer who had been entered into five phase II/III trials using fulvestrant as first- to ninth-line endocrine therapy in our Unit since 1993, 54 patients who fulfilled the following criteria were studied for their subsequent endocrine response: (i) oestrogen receptor positive or unknown; (ii) having been on a subsequent endocrine therapy for $\geq 6$ months unless the disease progressed before; and (iii) with disease assessable for response according to International Union Against Cancer criteria. Eleven patients had received an aromatase inhibitor prior to fulvestrant, which resulted in five CBs (clinical benefit=objective remission/stable disease (SD)) for $\geq 6$ months). Twenty-eight patients achieved CB on fulvestrant. They went on subsequent endocrine therapy with two partial responses, 11 SDs and 15 PDs (progressive disease) at 6 months. The median survival from starting fulvestrant and subsequent endocrine therapy was respectively 46.6 and 18.2 months. Among the remaining 26 patients who progressed at 6 months on fulvestrant, there were three SDs and 23 PDs at 6 months on subsequent endocrine therapy. The median survival from starting fulvestrant and subsequent endocrine therapy was respectively 12.5 and 9.3 months. Of all these 54 patients, $30 \%$ $(n=16)$ therefore achieved CB using another (second- to tenth-line) endocrine agent (anastrozole= 26; tamoxifen=12; megestrol acetate $=11$; others $=5$ ). It would thus appear that further endocrine response can be induced in a reasonable proportion of patients after failing fulvestrant.
\end{abstract}

Endocrine-Related Cancer (2006) 13 251-255

\section{Introduction}

Endocrine therapies, such as tamoxifen and the aromatase inhibitors, are currently used for the treatment of hormone-sensitive advanced breast cancer, and lead to tumour regression or disease stabilisation in approximately $70 \%$ of patients when used upfront. While most tumours become resistant to initial endocrine therapy, many retain hormone sensitivity and respond to subsequent endocrine treatments. This sequential use of endocrine therapies may offer marked quality of life advantages by delaying the need for cytotoxic chemotherapy in some patients. Fulvestrant is the first in a novel class of anti-oestrogens - an oestrogen-receptor down-regulator (Wakeling et al. 1991, Robertson et al. 2001). Fulvestrant is effective at inhibiting the growth of breast cancer cells in in vitro and in vivo models of tamoxifen resistance (Osborne et al. 1994, 1995). Phase I/II clinical studies in postmenopausal women with advanced breast cancer who have progressed following tamoxifen therapy indicate that fulvestrant is active and well tolerated (Howell et al. 1995, 1996, Cheung et al. 2001). Recently reported phase III data have led to 
Table 1 Clinical trials from which patient data were analysed in this study

\begin{tabular}{ll}
\hline Trial & \multicolumn{1}{c}{ Description } \\
\hline 1 & $\begin{array}{l}\text { An open, phase II study in which patients progressing on tamoxifen therapy were treated with a monthly i.m. injection of } \\
\text { fulvestrant } 250 \mathrm{mg}\end{array}$ \\
$2 \quad \begin{array}{l}\text { An open, randomised, multicentre, phase III study designed to compare the efficacy and tolerability of a monthly i.m. } \\
\text { injection of fulvestrant } 250 \mathrm{mg} \text { with a once-daily oral dose of anastrozole } 1 \mathrm{mg} \text { in patient who had relapsed or progressed } \\
\text { following previous endocrine therapy } \\
\text { A phase III, double-blind, randomised, multicentre, comparative study of the efficacy and tolerability of a monthly i.m. } \\
\text { injection of fulvestrant } 250 \mathrm{mg} \text { with a once-daily oral dose of tamoxifen } 20 \mathrm{mg} \text { as first-line therapy for advanced breast } \\
\text { cancer } \\
\text { A randomised pharmacokinetic study comparing the pharmacokinetic profile of fulvestrant } 250 \text { mg given as a single } \\
5 \text { ml i.m. injection or as two } 2.5 \mathrm{ml} \text { i.m. injections } \\
\text { An open phase II study of the in vivo biological effects of fulvestrant in either elderly patients with primary breast cancer } \\
\text { or patients with locally advanced breast cancer in whom primary endocrine therapy is the treatment of choice }\end{array}$ \\
\hline
\end{tabular}

licensing of fulvestrant for clinical use in Europe and the United States (Howell et al. 2002, Osborne et al. 2002, Robertson et al. 2003).

In our centre we have participated in five phase II/ III clinical trials which have examined the activity of fulvestrant in postmenopausal women with advanced breast cancer (Table 1). Here we report a retrospective analysis of subsequent therapy data from these trials to evaluate the effects of further endocrine therapies in patients who received fulvestrant in our centre.

\section{Patients and methods}

The objective of the study was to obtain retrospective data from patients in all the above-mentioned clinical trials enrolled at Nottingham City Hospital to evaluate whether tumour responses to subsequent endocrine therapy were seen in patients who initially showed clinical benefit (CB) on fulvestrant and in those who failed to show such benefit.

All trials recruited postmenopausal women (defined as fulfilling any of the following criteria: aged $\geq 60$ years; aged $\geq 45$ years with amenorrhoea $>12$ months and an intact uterus; follicle-stimulating hormone levels within postmenopausal range; having undergone a bilateral oophorectomy) with breast cancer not considered amenable to curative treatment, a life expectancy of $\geq 3$ months and a WHO performance status of $\leq 2$.

Patients had to fulfil the following inclusion criteria in this retrospective analysis: (i) have a positive or unknown oestrogen receptor (ER) status; (ii) be in receipt of endocrine therapy following prior fulvestrant for $\geq 6$ months (British Breast Group 1974) except for those who progressed within 6 months; and (iii) have disease assessable for response according to criteria laid down by the International Union Against Cancer (UICC) (Hayward et al. 1977).

All patients received fulvestrant (AstraZeneca) $250 \mathrm{mg}$ i.m. every $28 \pm 3$ days, given predominantly by one breast-care research nurse ( $\mathrm{RO})$. All patients in this study continued treatment until disease progression. Upon disease progression they undertook further therapy as determined by one of the two clinicians (K L C and JF R R). Patients were followed up in the same breast cancer clinic and monitored for progression and survival until death. The responses to treatment with fulvestrant and subsequent treatment were assessed according to UICC criteria. Objective response (OR) was defined as complete response (CR) or partial response (PR). CB was defined as OR, or stable disease (SD) $\geq 24$ weeks (Robertson et al. 1997).

\section{Results}

Fifty-four patients (mean age at the time of starting fulvestrant 67 years, range 33-90 years) fulfilled the selection criteria for inclusion in this analysis, with most patients $(83 \%)$ having received fulvestrant as first- $(n=18)$ or second- $(n=27)$ line endocrine therapy. The remaining nine patients had fulvestrant as third- $(n=6)$, fourth- $(n=1)$, fifth- $(n=1)$ and ninth$(n=1)$ line endocrine therapy. The majority of the tumours were ER positive $(n=49)$, with only five having unknown ER status.

Eleven patients had received an aromatase inhibitor prior to fulvestrant and five patients subsequently derived CB with fulvestrant (Table 2).

Twenty-eight patients $(52 \%)$ derived $\mathrm{CB}$ from fulvestrant. The best responses to fulvestrant in all 54 patients were CR $(n=1), \mathrm{PR}(n=13)$ and SD $(n=20)$. The median time to progression (TTP) on fulvestrant for all patients was 9.3 months (range 
Table 2 Response at 6 months (number of patients) to fulvestrant in patients who had received prior treatment with an aromatase inhibitor

\begin{tabular}{lcccc}
\hline & PR & SD & PD & Total \\
\hline $\begin{array}{l}\text { Patients who had received } \\
\text { prior to fulvestrant }\end{array}$ & & & & \\
Anastrozole & 2 & 2 & 3 & 7 \\
Exemestane & 0 & 1 & 0 & 1 \\
All therapies & 2 & 3 & 3 & 8 \\
Patients who had received aromatase inhibitors as the second \\
treatment prior to fulvestrant \\
$\begin{array}{l}\text { Anastrozole } \\
\text { Formestane }\end{array}$ \\
All therapies & 0 & 0 & 2 & 2 \\
\hline
\end{tabular}

$\mathrm{PR}$, partial response; SD, stable disease; PD, progressive disease.

1-75 months). All patients subsequently received endocrine therapy consisting of anastrozole (AstraZeneca) $(n=26)$, tamoxifen $(n=12)$, megestrol acetate (Bristol-Myers Squibb) $(n=11)$, exemestane (Pfizer) $(n=3)$, ethinyloestradiol $(n=1)$ or 'withdrawal' therapy (i.e. discontinuation of an endocrine agent aiming to induce a withdrawal response) $(n=1)$. Such subsequent endocrine therapy resulted in two PRs, 14 SDs (i.e. $30 \% \mathrm{CB}$ rate) and 38 progressive diseases (PDs) with a median TTP of 5.7 months (range 1-60 months). The median survival of all patients calculated from starting fulvestrant and from starting the subsequent endocrine therapy was respectively 25.9 and 13.7 months.

The 28 patients who had derived CB from fulvestrant subsequently received different endocrine agents resulting in $13 \mathrm{CBs}(46 \%)$ (Table 3 ). The median survival of these 28 patients calculated from starting fulvestrant and from starting the subsequent endocrine therapy was respectively 46.6 and 18.2 months.

The remaining 26 patients who had not derived CB from fulvestrant subsequently received different endocrine agents resulting in three CBs $(12 \%)$ (Table 3 ). The median survival of these 26 patients calculated from starting fulvestrant and from starting the subsequent endocrine therapy was respectively 12.5 and 9.3 months.

\section{Discussion}

Data from two prospective multicentre randomised trials comparing 4-weekly fulvestrant $250 \mathrm{mg}$ i.m. injections $(n=423)$ with daily anastrozole $1 \mathrm{mg}$ orally $(n=423)$ for postmenopausal women with hormonesensitive advanced breast cancer progressing on tamoxifen showed equivalent efficacy, leading to
Table 3 Response at 6 months (number of patients) to subsequent endocrine therapy in patients who received fulvestrant

\begin{tabular}{lcccc}
\hline & PR & SD & PD & Total \\
\hline Patients who derived clinical & benefit & from & fulvestrant & \\
Tamoxifen & 0 & 4 & 4 & 8 \\
Anastrozole & 2 & 6 & 4 & 12 \\
Exemestane & 0 & 0 & 1 & 1 \\
Megestrol acetate & 0 & 1 & 4 & 5 \\
Ethinyloestradiol & 0 & 0 & 1 & 1 \\
Withdrawal therapy & 0 & 0 & 1 & 1 \\
All therapies & 2 & 11 & 15 & 28 \\
Patients who failed to derive clinical & benefit & 0 & 4 & 4 \\
Tamoxifen & 0 & 0 & 4 & fulvestrant \\
Anastrozole & 0 & 2 & 12 & 14 \\
Exemestane & 0 & 0 & 2 & 2 \\
Megestrol acetate & 0 & 1 & 5 & 6 \\
All therapies & 0 & 3 & 23 & 26
\end{tabular}

$\mathrm{PR}$, partial response; SD, stable disease; $\mathrm{PD}$, progressive disease.

licensing of fulvestrant as a second-line endocrine therapy (Howell et al. 2002, Osborne et al. 2002, Robertson et al. 2003). One of the current and future clinical and research questions is on the optimal sequencing, given the number of potentially effective endocrine agents currently available. There are a number of important factors when sequencing of endocrine agents is considered. First, there should be a lack of cross-resistance among these agents due to different mechanisms of action. Secondly, both efficacy and tolerability benefits need to be looked at, with the most effective agent being used first. This should, however, allow maintenance of activity to subsequent endocrine therapy. The study reported in this paper aimed to find out, from the experience of a single centre, the activity of other endocrine agents following fulvestrant.

While the randomised trials showed efficacy of fulvestrant following tamoxifen, another important question relating to optimal sequencing is whether fulvestrant and other endocrine agents remain effective following an aromatase inhibitor. This is especially important given the recent data showing superiority of the third-generation aromatase inhibitors over tamoxifen as an adjuvant endocrine therapy. It can therefore be envisaged that fulvestrant, and other endocrine agents, will need to be used following progression on an aromatase inhibitor. The results from the study reported here have also shed some light on this question, confirming that fulvestrant remains effective following prior treatment with an aromatase inhibitor (Table 2). 
In the randomised trials using fulvestrant as second-line endocrine therapy following tamoxifen, a questionnaire survey to investigators from different participating centres demonstrated responsiveness to further endocrine therapy (predominantly aromatase inhibitors) (Vergote et al. 2003). Among the 54 patients who derived CB from fulvestrant, subsequent endocrine therapy resulted in four PRs, 21 SDs and 29 PDs at 6 months $(\mathrm{CB}$ rate $=46 \%)$. Data were also available from 51 patients who progressed on fulvestrant and who received further endocrine therapy, achieving one PR, 17 SDs and 33 PDs (CB rate $=35 \%$ ). Since the early 1990s, we have been taking part in most of the clinical trials (phase II/III) involving fulvestrant. Therefore, data from this paper support findings in the above study. It has the additional strength of obtaining trial data from a single centre. Furthermore, it has also demonstrated the responsiveness of further endocrine therapy after fulvestrant had been used, not only as second-line agent as described by Vergote et al., but as a first- to ninth-line agent, showing CB rates of 46 and $12 \%$ respectively in the groups who derived or did not derive $\mathrm{CB}$ on fulvestrant. The result certainly adds to the knowledge in sequencing endocrine therapies.

Furthermore, the prolonged survival following the use of fulvestrant and subsequent endocrine therapy is impressive, especially in the group who derived CB from fulvestrant (median being 46.6 and 18.2 months respectively).

In addition to demonstrating efficacy benefits, tolerability is an important factor in sequencing different agents. Fulvestrant, the only pure anti-oestrogen available for clinical use thus far, is unique in that it is the only endocrine agent administered i.m. Parenteral administration may have potential advantages in ensuring compliance and maintaining useful contacts with healthcare personnel, which may be reassuring, although i.m. injections have their limitations in patients with bleeding diathesis. Personal experience (Owers 2004) has not demonstrated significant problems with this route of administration and quality of life has been shown to be equivalent in both arms of patients receiving fulvestrant and anastrozole in the randomised trial mentioned above (Howell et al. 2002).

While fulvestrant is a potent pure anti-oestrogen, it has not yet been shown to down-regulate the ER to a negligible level (Robertson et al. 2001). It therefore remains uncertain as to whether the addition of an aromatase inhibitor (which produces oestrogen deprivation and clinically superior efficacy over tamoxifen) to fulvestrant will further improve efficacy without significantly worsening the side-effect profile. Such a combination approach is currently one of the key research questions being addressed by ongoing clinical trials. These trials compare fulvestrant with one of the third-generation aromatase inhibitors, and with their combination, in the pre-surgical setting for operable primary breast cancer, as a first-line endocrine therapy or after failing a non-steroidal aromatase inhibitor for hormone-sensitive postmenopausal advanced breast cancer.

In summary, clinical experience from our centre suggests that patients who respond to fulvestrant and then progress may retain sensitivity to subsequent endocrine therapy. Disease control with subsequent endocrine agents was also seen in some patients who did not respond to fulvestrant therapy. This does not apply only after fulvestrant has been used as first- or second-line therapy, but also after it has been used as a further endocrine agent. Fulvestrant provides an additional treatment option for advanced breast cancer following progression on prior endocrine therapy.

\section{Acknowledgements}

The authors declare that there is no conflict of interest that would prejudice the impartiality of this scientific work.

\section{References}

British Breast Group 1974 Assessment of response to treatment in advanced breast cancer. Lancet 2 38-39.

Cheung KL, Owers R, Howell A \& Robertson JFR 2001

Survival updates of a phase II study comparing long-acting ICI 182,780 (Faslodex) with megestrol acetate as second-line endocrine therapy for breast cancer. Proceedings of 7 th Nottingham International Breast Cancer Conference Page 21.

Hayward JL, Carbone PP, Heuson JC, Kumaoka S, Segaloff A \& Rubens RD 1977 Assessment of response to therapy in advanced breast cancer. Cancer 39 1289-1294.

Howell A, DeFriend D, Robertson JFR, Blamey RW \& Walton P 1995 Response to a specific pure anti-oestrogen (ICI 182,780) in tamoxifen resistant breast cancer. Lancet 345 29-30.

Howell A, DeFriend DJ, Robertson JFR, Blamey RW, Anderson L, Anderson E, Sutcliffe FA \& Walton P 1996 Pharmacokinetics, pharmacological and anti-tumour effects of the specific anti-oestrogen ICI 182,780 in women with advanced breast cancer. British Journal of Cancer $\mathbf{7 4}$ 300-308.

Howell A, Robertson JFR, Quaresma Albano J, Aschermannova A, Mauriac L, Kleeberg UR, Vergote I, Erikstein B, Webster A \& Morris C 2002 Fulvestrant, formerly ICI 182,780, is as effective as anastrozole in postmenopausal women with 
advanced breast cancer progressing after prior endocrine treatment. Journal of Clinical Oncology 20 3396-3403.

Osborne CK, Jarman M, McCague R, Coronado EB, Hilsenbeck SG \& Wakeling AE 1994 The importance of tamoxifen metabolism in tamoxifen-stimulated breast tumour growth. Cancer Chemistry and Pharmacology 34 89-95.

Osborne CK, Coronado-Heinsohn EB, Hilsenbeck SG, McCue BL, Wakeling AE, McClelland RA, Manning DL \& Nicholson RI 1995 Comparison of the effects of a pure steroidal anti-oestrogen with those of tamoxifen in a model of human breast cancer. Journal of the National Cancer Institute 87 746-750.

Osborne CK, Pippen J, Jones SE, Parker LM, Ellis M, Come S, Gertler SZ, May JT, Burton G, Dimery I et al. 2002 Double-blind, randomized trial comparing the efficacy and tolerability of fulvestrant versus anastrozole in postmenopausal women with advanced breast cancer progressing on prior endocrine therapy: results of a North American trial. Journal of Clinical Oncology 20 3386-3395.

Owers R 2004 Clinical experience with fulvestrant ('Faslodex'): a nurse's perspective. European Journal of Oncological Nursing 8 (Suppl 2) S89-S94.

Robertson JFR, Willsher PC, Cheung KL \& Blamey RW 1997 The clinical relevance of static disease (no change) category for 6 months on endocrine therapy in patients with breast cancer. European Journal of Cancer 33 1774-1779.

Robertson JFR, Nicholson RI, Bundred NJ, Anderson E, Rayter Z, Dowsett M, Fox JN, Gee JM, Webster A, Wakeling AE et al. 2001 Comparison of the short-term biological effects of 7alpha-[9-4,4,5,5,5pentafluoropentylsulfinyl(-nonyl]estra-1,3,5,(10)triene-3,17beta-diol (Fulvestrant) versus tamoxifen in postmenopausal women with primary breast cancer. Cancer Research 61 6739-6746.

Robertson JF, Osborne CK, Howell A, Jones SE, Mauriac L, Ellis M, Kleeberg UR, Come SE, Vergote I, Gertler S et al. 2003 Fulvestrant versus anastrozole for the treatment of advanced breast carcinoma in postmenopausal women: a prospective combined analysis of two multicenter trials. Cancer 98 229-238.

Vergote I, Robertson JFR, Kleeberg U, Burton G, Osborne CK \& Mauriac L; Trial 0020 Investigators; Trial 0021 Investigators 2003 Postmenopausal women who progress on fulvestrant ('Faslodex') remain sensitive to further endocrine therapy. Breast Cancer Research and Treatment 79 207-211.

Wakeling AE, Dukes M \& Bowler J 1991 A potent specific pure anti-oestrogen with clinical potential. Cancer Research 51 3867-3873. 
